\title{
EBSD Observation for Reversible Behavior of Deformation Twins in AZ31B Magnesium Alloy
}

\author{
Tsuyoshi Uota, Takuya Suzu, Shinji Fukumoto and Atsushi Yamamoto \\ Graduate School of Engineering, University of Hyogo, Himeji 671-2201, Japan
}

A bending device used for SEM-EBSD systems has been developed by the authors, which enables one to intermittently observe changes in microstructures induced by deformation in the same viewing area. Alternate compression-tension loading was applied to a sheet specimen of AZ31B magnesium alloy by bending the specimen. Twins were formed by compression straining. They remained after un-loading. Applying tensile straining lead to the disappearance of all the twins. Most of twins were those of $\{10 \overline{1} 2\}$ types. Anisotropy in mechanical properties of magnesium alloys would be affected by this twinning and de-twinning behaviors. [doi:10.2320/matertrans.M2009165]

(Received May 11, 2009; Accepted June 10, 2009; Published July 15, 2009)

Keywords: deformation twin, magnesium alloy, compression deformation, detwinning

\section{Introduction}

Deformation twins play an important role in deformation with low cycle fatigue on magnesium alloys. Cold-rolled sheets or extruded bars show the basal plane texture with the $c$-axis perpendicular to the sheet surface or the longitudinal axis of the bars, respectively. When such the materials are deformed with compression in the direction perpendicular to the $c$-axis or with tension in the direction parallel to the $c$ axis, $\{10 \overline{1} 2\}$ twins are easily formed. ${ }^{1)}$ Therefore, in an alternate loading-unloading unidirectional deformation leads to anisotropic hysteresis loops as reported by Cáceres et al. ${ }^{2)}$ Somekawa et al. ${ }^{3)}$ reported that a number of twins were formed in AZ31 alloy by fatigue deformation with a stress ration of $R=-1$ and $R=0.1$ when the stress amplitude exceeded a yield stress in compression. They showed that $\{10 \overline{1} 2\}$ twins were mainly formed using an optical microscope and as well as a scanning electron microscope (SEM) equipped with an electron backscattered diffraction (EBSD) analyzer. They observed that the specimen fatigued after 100 cycles. It was not clear whether the number of twins gradually increased, or were all formed at once. In the latter case, twins are formed through compression straining and disappear during tensile straining. Wu et al. ${ }^{1)}$ carried out insitu neutron diffraction measurements during loading-unloading cycles on ZK60A alloy and concluded that twiningdetwining occurred on the basis of crystallographic consideration. They also showed an optical micrograph of twins formed by compression straining, but the micrograph was not obtained through an in-situ experiment. Cáceres et al. ${ }^{2)}$ carried out an in-situ observation using an optical microscope under loading-unloading conditions, and showed that the thickness of the twins increased or decreased depending upon the loading conditions, moreover, complete disappearance was also observed. However, there was lack of crystallographic data, because only an optical microscope was used for observation.

An in-situ observation using an SEM-EBSD method is the optimum method to clarify twin formation in both metallographic and crystallographic meanings and formations. In the present study, SEM-EBSD analyses are carried out on the same view field in an AZ31 alloy sheet during compressiontension alternate straining.

\section{Experimental Procedures}

A small bending test device has been developed by the authors, which enables one to observe changes in microstructures in an SEM-EBSD system. Schematic illustrations of the device and specimen sizes are shown in Fig. 1. The whole sizes of the device are about $10 \times 40 \times 50 \mathrm{~mm}$, which can be attached on a standard sample holder for EBSD measurements. The specimen is alternately bent by using a micrometer-head or a screw. Displacement of the moving end of the specimen is measured by the micrometer-head to calculate the strain induced on the observed surface.

Specimens were prepared from a commercial AZ31B magnesium alloy sheet with $1.8 \mathrm{~mm}$ in thickness. The specimen was solution heat treated at $673 \mathrm{~K}$ for $3.6 \mathrm{ks}$. A surface for observation was mechanically polished and finished by etching using a solution of nitric acid: acetic acid : ethanol $:$ water $=5: 15: 20: 60{ }^{4}$ )

Micro-Vickers indentations were applied to the specimen surface for use as landmarks. The device with the specimen was set in an SEM, and EBSD on the solution heat treated condition was measured. The SEM was then leaked and compression strain was applied by manually turning the

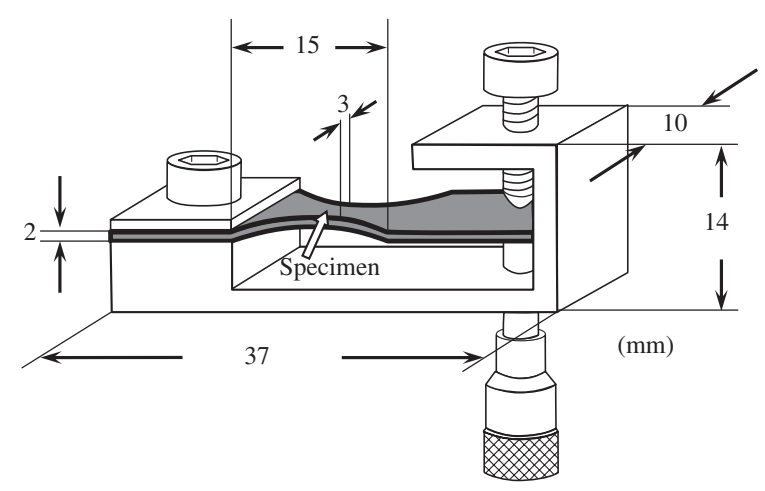

Fig. 1 Schematic illustration of the bending device and specimen with sizes. 

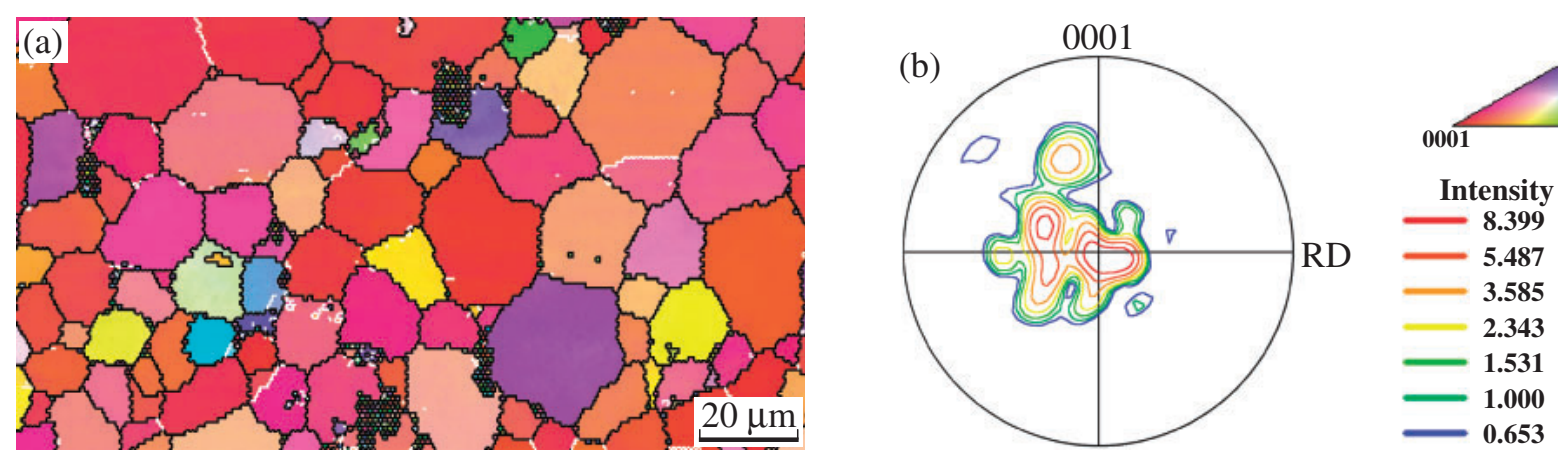

Fig. 2 IPF map of the specimen before bending deformation (a), and 0001 pole figure (b).
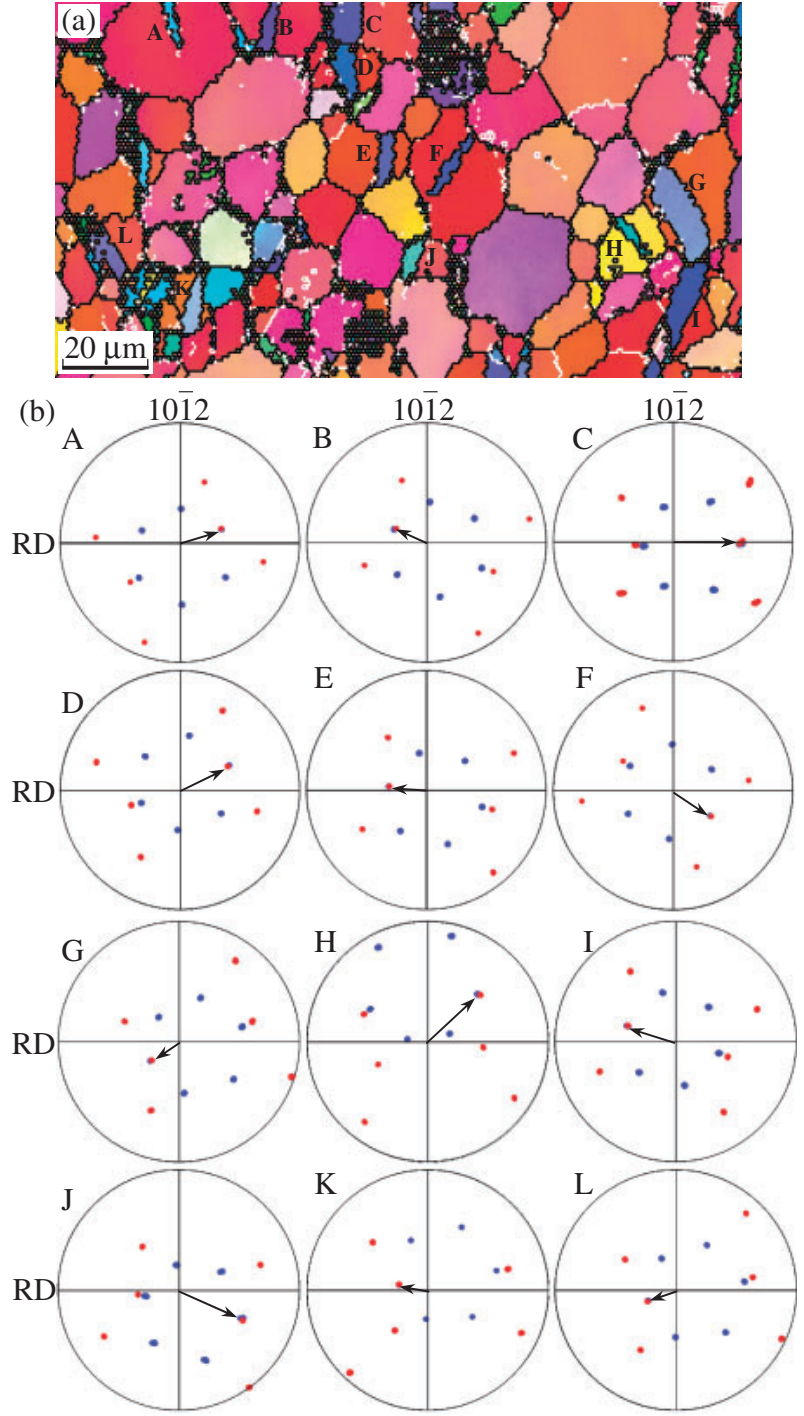

Fig. 3 (a) IPF map of the specimen under compression straining. (b) $10 \overline{1} 2$ pole figures for matrices and twins in the grains labeled $\mathrm{A}$ to $\mathrm{L}$ in (a). The blue and red solid circles indicate $10 \overline{1} 2$ poles for matrices and twins, respectively. The black arrows in (b) indicate the coincident $10 \overline{1} 2$ poles between the twins and the matrices, and also indicate the normals for the twin interfaces.

micrometer-head followed by a setting, and the SEM was again evacuated for measurements of the EBSD. These same procedures were repeated for unloading, loading tension, and re-unloading.
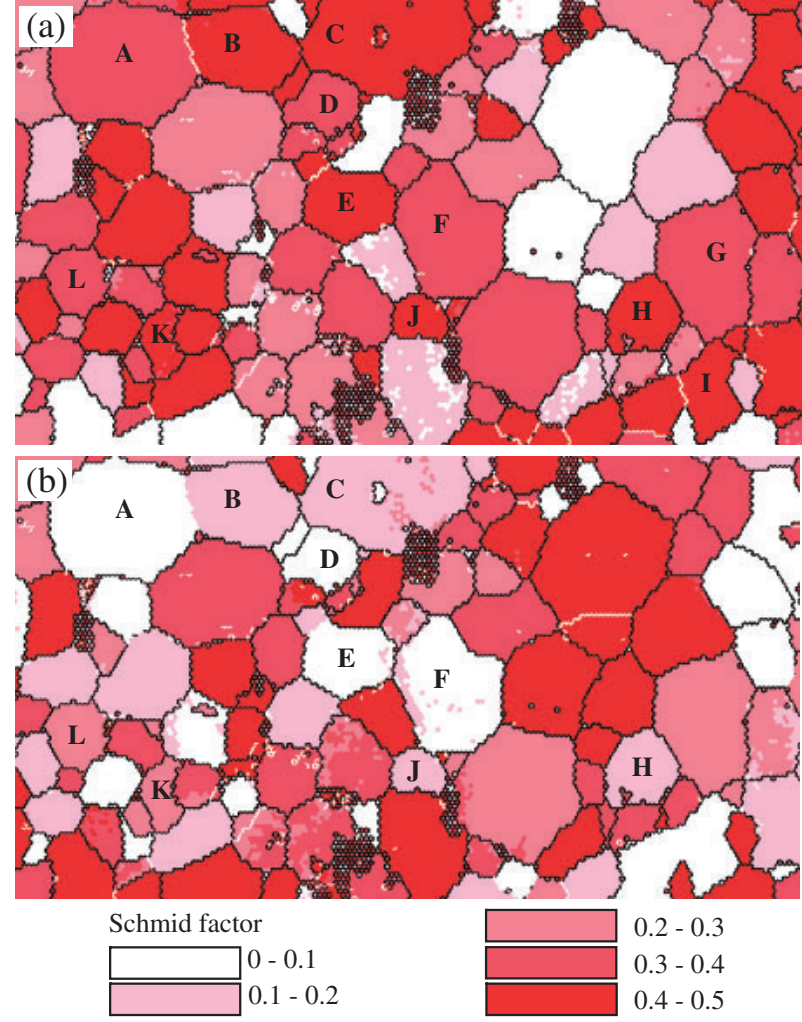

Fig. 4 Schmid factor maps for $\{10 \overline{1} \overline{2}\},\langle 10 \overline{1} 1\rangle$ twins and $\{0001\},\langle 1 \overline{2} 10\rangle$ slip, (a) and (b), respectively.

\section{Results and Discussion}

An inverse pole figure (IPF) map for the solution heat treated specimen, before deformation, is shown in Fig. 2(a). Although the specimen had a typical basal plane texture, the intensity maxima were dispersed as shown in Fig. 2(b), due to the small number of grains in the viewing area.

When the specimen was bent in order to apply compression strain, many twins were formed, as shown in Fig. 3(a). Orientation relationships between the twins and the mother grains labeled A to L are shown in Fig. 3(b) with $\{10 \overline{1} 2\}$ pole figures. The blue solid circles indicate the $\{10 \overline{1} 2\}$ poles in the mother grain, while the red solid circles indicate those in the twins. The arrows indicate coincident $\{10 \overline{1} 2\}$ poles between the mother grains and the twins. The arrows also show the normals for the twin interfaces, therefore they are perpen- 

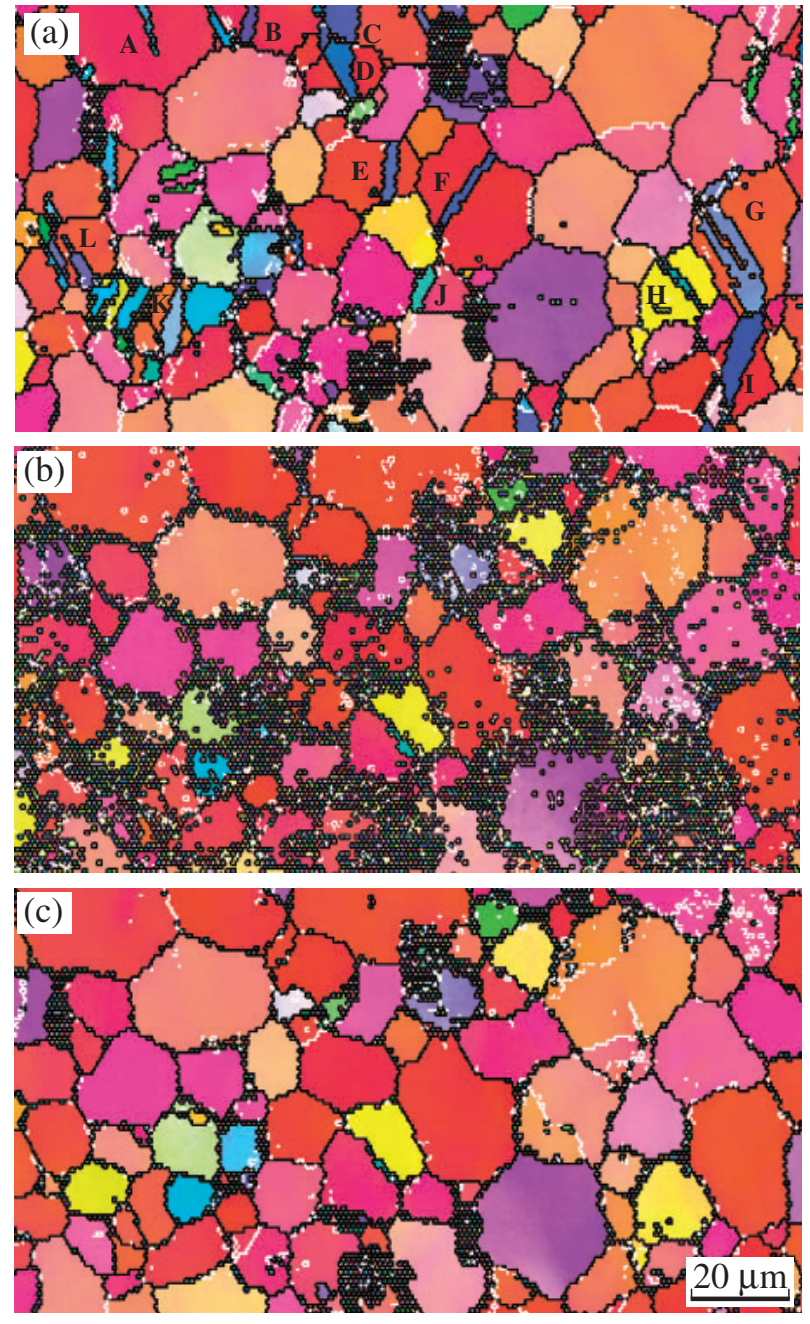

Fig. 5 IPF maps taken of the same areas shown in Figs. 2 and 3, under unloading after compression (a), under tension (b), and re-unloading (c).

dicular to those observed in the IPF map. All the twins are $\{10 \overline{1} 2\}$ type.

Schmid factor maps for $\{10 \overline{1} \overline{2}\},\langle 10 \overline{1} 1\rangle$ twins and $\{0001\}$, $\langle 1 \overline{2} 10\rangle$ slip are shown in Figs. 4(a) and (b), respectively, in which the solution heat treated image shown in Fig. 2 was used. The grains in which the twins were formed are labeled A to L, as shown in Fig. 3(a). The twins were formed in such grains with a higher Schmid factor for a twin, and a lower Schmid factor for basal slip.
IPF maps subsequently obtained under unloading, tension loading and re-unloading are shown in Figs. 5(a), (b) and (c), respectively. Twins were remained under unloading condition (a), disappeared under tension loading (b) and did not appear under re-unloading (c). The reason why the quality of the IPF map decreased under tensile conditions has not been clarified. This is not a matter for the present study. All the twins formed under compression completely disappeared under tensile loading.

In the case of a unidirectional deformation, localization of strain would occur at the twin boundary and lead to failure, as Koike pointed out. ${ }^{5)}$ Under an alternate compression-tension straining, twins formed by compression could be reversed into a mother lattice through tension, as shown in the present study. Therefore, accumulation of strain due to twining would not be so significant, at least at the early stage of alternating. On the other hand, the present results also showed that plastic deformation in tensile straining is mainly compensated by dislocation motions. Reversible behavior of dislocations is not expected to occur during stress reversion. When the stress is reversed again into compression, twins are formed in the areas containing dislocations. Whether or not such twins containing dislocations can be reversed is an important subject that should be clarified.

\section{Summary}

Intermittent observations of changes in microstructures during alternate compression-tension straining have been carried out on the same viewing area of an AZ31B magnesium alloy by using a bending deformation device used for SEM-EBSD system. Twins are formed under compression straining, remain after un-loading, but completely disappear under tensile straining.

\section{REFERENCES}

1) L. Wu, A. Jain, D. W. Brown, G. M. Stoica, S. R. Agnew, B. Clauser, D. E. Fielden and P. K. Liaw: Azta Mater. 56 (2008) 699-695.

2) C. H. Caceres, T. Sumitomo and M. Veidt: Acta Mater. 51 (2003) 6211-6218.

3) H. Somekawa, N. Maruyama, S. Hiromoto, A. Yamamoto and T. Mukai: Mater. Trans. 49 (2008) 681-684.

4) M. R. Barnett, M. D. Nave and C. J. Bettles: Mater. Sci. Eng. A 386A (2004) 205-211.

5) J. Koike: Metall. Mater. Trans. A 36A (2005) 1689-1696. 